\title{
$\mathrm{X}$.
}

\section{Beiträge zur Lehre von der fieberhaften (durch pyrogene Substanzen bewirkten) Temperaturerhöhung von}

\author{
v. Dubczcanski und B. Naunyn \\ in Bern. \\ in Königsberg.
}

\section{Hierzu Tafel VI.}

Durch die Untersuchungen ron Tscheschichin*), Naunyn and Quincke**), Heidenhain and seinen Schülern***) ist es festgestellt, dass durch Verletzungen des Centralnervensystems Steigerungen der Körpertemperatur über das Normale herbeigeführt werden können. Tscheschichin beobachtete bei einem Kaninchen, bei welchem er durch Schnitt den Pons Varolii von der Medulla oblongata getrennt, eine bedeutende Steigerung der Körpertemperatur. Seine Versuche wurden von Heidenhain wiederholt. Heidenhain fand, dass die fragliche Temperatursteigerung nicht nur nach Trennung des Pons vou der Medulla oblongata, sondern auch dann zu Stande kommt, wenn Nadeln in den untern Rand des Pons oder den obern Rand der Medulla oblongata eingeführt werden; er schloss hieraus, dass dieselbe nicht durch Lähmung, sondern dureh Reizung von die Temperatur des Körperss regulirenden Centren oder

*) Tschescinichin, Zur Lehre von der thierischẹn Wärme. Reichert and D n bois's Archiv 1866 pag. 170.

**) Na unyn und Quinc ke, Ueber den Finfluss des Centralnervensystems auf die Wärmebildung; 1. Abhandling Reichert und Dubois's Archiv 1869 pag. 174. 2. Abhandlung ibid. 1869 pag. 521.

***) Versuche über den Einfluss gewisser Hirntheile auf die Temperatur u s. w., mitgetheilt ron R. Heidenhain. Pflüger's Archiv 1970 pag. 578. 
Fasern bedingt sei und suchte diese Anschaung durch directe Erregung jener Hirntheile mittelst elektrischer Reizung zu erlärten.

Die Resultate dieser Versuche fielen der Art aus, dass Heiden. hain ihnen eine lediglich „subjectiv überzengende" Kraft beimisst. Naunyn und Quincke gingen bei Anstellung ihrer Versuche von einer Ueberlegung aus, welche ihnen andere von Tscheschichin, Schiff u. Anderen gemachte Beobachtungen und ältere klinische Erfahrungen an die Hand gaben; es ist dies folgende:

Ruickenmarksdurchtrennungen führen meist zu erheblichem Sinken der Körpertemperatur, namentlich bei kleinen Thieren (Kaninchen, auch kleineren Hunden); hier gelingt $t^{*}$ es, das Sinken der Körpertemperatur durch Einwickelung der Thiere in Watte aufzuhalten. Bei grossen Organismen (Menschen) wird verhältnissmässig häufig nach acuter (traumatischer) Continuitätstrennung des Rückenmarkes Steigerung der Körpertemperatur bis zu den höchsten Graden beobachtet; leichter, wie es scheint dann, wenn die den Kranken umgebende Atmosphäre selbst eine hohe Temperatur zeigt.

Angenommen nun: es würden durch die Continuitätstrennung des Riuckenmarks in den Thieren einmal die Bedingungen fur die Wärmeabgabe, d. h. die Abkïhlung, andererseits die Bedingungen für die Wärmeproduction, d. h. die Erwärmung derselben gesteigert, so ist dies leicht begreiflich: denn

a) die Wärmeabgabe ist offenbar eine Function der KörperoberHäche, die Wärmeproduction eine solche innerer Organe; es wird daher, eine gleichzeitige Steigerung der Wärmeproduction und der Wärmeabgabe als Folge der Ruickenmarksdurchtrennung vorausgesetzt, das Resultat dieser beiden Factoren um so eher eine Steigerung der Körpertemperatur (d. h. ein Ueberwiegen der Wärmeproduction) sein, wenn die Oberfläche der Thiere im Verhältniss zur Masse ihrer inneren Organe klein ist, $d$. h. wenn die Thiere möglichst gross sind. Umgekehrt bei kleinen Thieren, bei welchen die Körperoberfläche im Verhältniss zur Masse der innern Organe gross ist, wird leichter ein Ueberwiegen der Wärmeabgabe, d. h. ein Sinken der Körpertemperatur die Folge sein.

b) Selbstvertändlich ist es dann ausserdem von besonderer Wichtigkeit für den Erfolg des Eingriffs, ob die äusseren Bedingungen fürr die Wärmeabgabe vom Körper günstig sind, d. h. hauptsächlich wenigstens, ob die umgebende Atmosphäre kalt oder warm ist etc.

Die Resultate der Versuche von N. und Q. sind in jeder Richtung geeignet, die Richtigkeit der ron ihnen entwickelten Anschauung $\mathrm{zu}$ beweisen. 
5
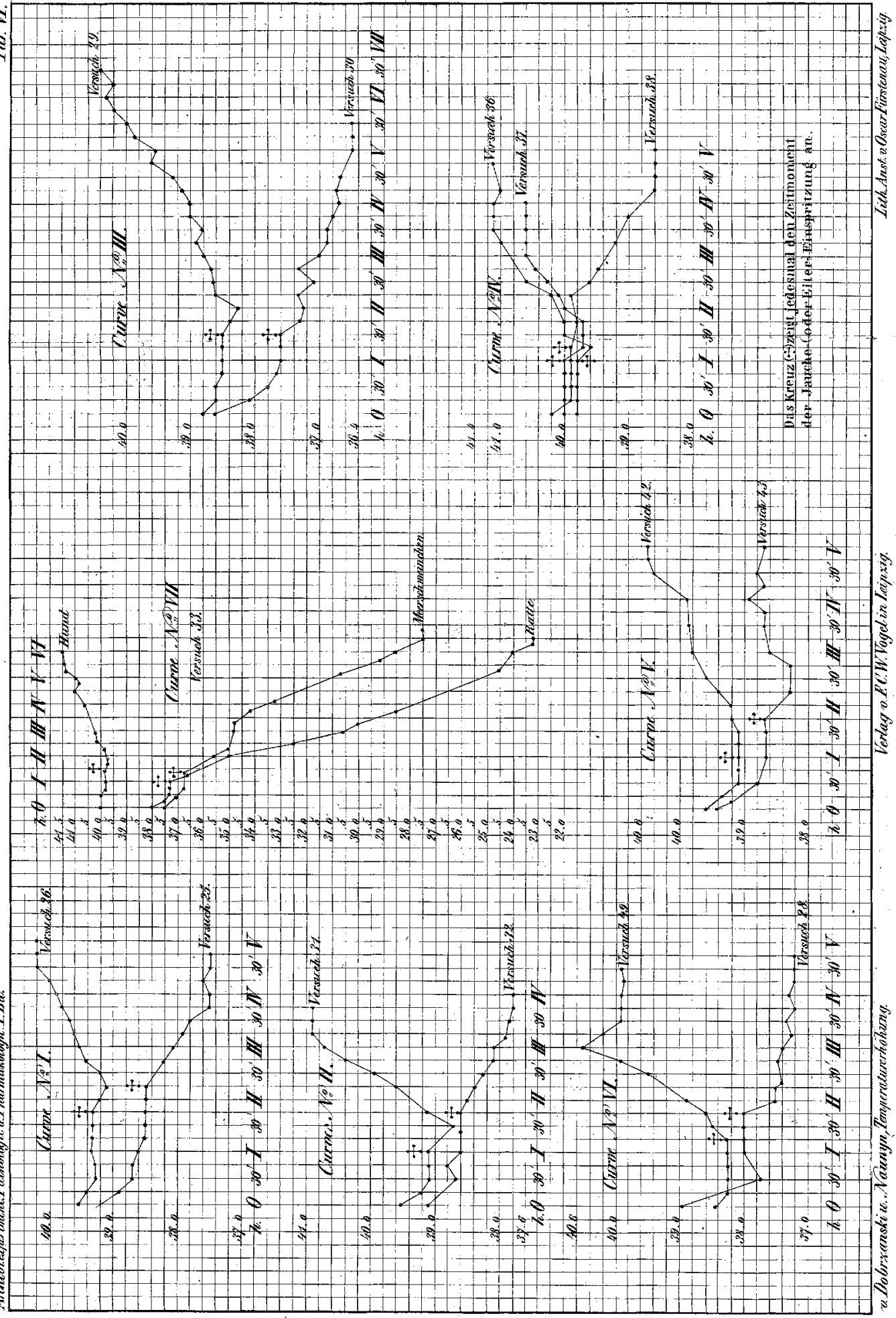
Sie wiesen nach, dass

1. auch bei kleineren Thieren (kleineren oder mittelgrossen Hunden) Rückenmarksquetschung im unteren Halstheil constant nicht Sinken, sondern sogar sehr bedeutende Steigerung der Körpertemperatur bewirkt, wenn die Wärmeabgabe durch Einführung der Thiere in einen warmen Raum gehemmt wird, in welchem, wie Controllversuche zeigten, Thiere mit normalem Riickenmarke sich viele Stunden aufhalten konnten, ohne eine Steigerung ihrer Körpertemperatur zu erfahren.

2. dass sich das Gleiche, das Steigen der Körpertemperatur der Thiere, nach einer Operation ebenso constant auch ohne Anwendung einer künstlichen Erwärmung bei einer mittleren Temperatur der Atmosphäre dann einstelit, wenn die Thiere so ausgewählt werden, dass bei ihnen an und für sich die unter a) angeführten für das Ueberwiegen der Wärmeproduction guinstigen Bedingungen erfüllt sind, d. h. wenn man den Versuch an möglichst grossen Thieren (recht grossen Hunden) anstellt.

Hierans schlossen N. und Q., dass im Ritekenmarke neben den Fasern, welche einen regulirenden Einfluss auf die Wärmeabgabe ausibben, auch solche Nervenfasern verlaufen, welche einen regulirenden (hemmenden) Einfluss auf die Wärmebildung vermitteln; lediglich so sei die trotz der gesteigerten Wärmeabgabe unter den angeführten Bedingungen nach der Riuckenmarksdurchschneidung constant auftretende Steigerung der Körpertemperatur zu erklären.

$\mathrm{Ob}$ diese fraglichen Nervenfasern identisch mit denen seien, welche die Innervation für die Gefässe der inneren Organe vermitteln (vasomotorische Nervenfasern) oder nicht - die Entscheidung dieser Frage liessen sie ausdriticklich vollkommen dahin gestellt. Gegen die Arbeit von N. und Q., den Schluss sowohl, wie gegen die Richtigkeit der mitgetheilten Thatsachen sind Einwände erhoben

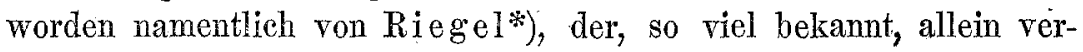
meintlich entgegenstehende Versuchsresultate genauer mittheilt.

Dieselben sind indessen in keiner Weise geeignet die Richtigkeit der von N. und Q. mitgetheilten Thatsachen und Schlïsse in Frage zu stellen.

*) Riegel, Ueber den Einfluss des Centralnervensystems auf die thierische Wärme. Pflüger's Archiv Bd. V. 1872 pag. 629. Die von Rosenthal gemachten Einwände (Zur Kenntniss der Wärmeregulirung. Erlangen 1872) sind ebensowenig stichhaltig. Sie sind zum grossen Theil schon von N. und Q. in ihrer mehrfach erwähnten Arbeit berücksichtigt und widerlegt, überhaupt aber einer eingehenden Kritik nicht zugängig, da die Versuche, auf welchen sie beruhen, nicht mitgetheilt sind. 
Es scheint zunächst, dass die zweite der beiden Abhandlungen, welche N. und Q. dem in Rede stehenden Gegenstande widmeten, Riegel vollkommen unbekannt geblieben ist; wenigstens findet sich in Riegel's umfangreicher Arbeit keinerlei Hinweis auf die in jener zweiten Abhandlung von N. und Q. mitgetheilten Versuche, welche eben den Beweis für das oben unter 2. Angeführte enthalten. Da es aber, wie dort gezeigt, gelingt die fragliche Temperatursteigerung nach Rückenmarksdurchtrennung in gewöhnlicher Atmosphäre bei mittlerer Temperatur constant zu erhalten, sofern man nur recht grosse Hunde zum Versuche auswählt, so verlieren Riegel's Einwürfe gegen die Richtigkeit jener Thatsache ihren Werth; denn dieselben stiitzen sich in der Hauptsache auf den Nachweis, dass Temperatursteigerung bei Thieren im warmen Raume auch ohne Rückenmarksdurchschneidung vorkommt.

Von dem Mitwirken eines hierauf beruhenden Versuchsfehlers in den Versuchen von $N$. und $Q$. kann aber da jedenfalls keine Rede sein, wo der warme Raum gar nicht angewendet wurde.

Was ibrigens die bezigglichen Versuche Riegel's im warmen Raume anlangt, so sind dieselben mit den ron N. und Q. angestellten nicht vergleichbar. Letztere wandten einen Raum an, der so temperirt und so beschaffen ist, dass, wie auch wiederholte Controllversuche lehrten, eine Steigerung der Körpertemperatur normaler Hunde in demselben nicht stathat. Riegel stellte die Versuche, welche ihn zur Behauptung des Gegentheils führten, mit narkotisirten Thieren an. Vorläufig aber ist es gewiss nicht ausgemacht, wie die Narkotisirung (durch Chloroform oder Morphium oder beides), der Rie gel seine Thiere unterwarf, auf dieselben im warmem Raume wirkt.

Dass hier die Herabsetzung der Respiration durch die Narcotica die entscheidende Rolle, die Riegel ihr zuschreibt, spielt, scheint namentlich bei Bericksichtigung der bekannten, unter Schmiedeberg's Leitung ansgefuhrten Arbeit Scheinesson's*) nicht wahrscheinlich. Jedenfalls ist die daran geknüpfte Behauptung Riegel's, dass bei Thieren die Haut rermöge der sie bedeckenden Haare ete. als Wärmeregulator nur eine untergeordnete Rolle spiele - nicht erwiesen. Im Gegentheil, je kleiner das Thier, desto wichtiger muss die Rolle der Haut als wä́rmeregulirendes Organ sein, denn tum so grösser ist die Körperoberfläche im Verhältniss zur Masse der inneren Organe.

Die Bedeckung der Haut der Thiere durch Haare ete. kann einen so selr erheblichen Unterschied ihrer Wärmeökonomie ron

*) Untersuchungen über den Einfluss des Chloroforms u. s. w. Dorpat 1868. 
der der Menschen wohl kaum bedingen; denn auch wir pflegen uns durch Bedeckungen ähnlicher Art vor iibermässiger Wärmeabgabe zu schiitzen.

Es musste den Einwürfen, welche gegen die Richtigkeit der von N. und Q. gefundenen Thatsachen und der darauf gebauten Schlüsse erhoben sind, hier eine eingehendere Besprechung gewidmet werden, da die im Nachfolgenden mitzutheilenden Versuche sich aufs Engste an das von jenen Festgestellte anschliessen. Wir hoffen übrigens, dass auch $\mathrm{Riegel}$ in demselben einen weiteren Beweis finden werde für die von ihm geläugnete Wichtigkeit der Haut als Wärmeregulator auch des Thierkörpers.

Es steht also nach dem bisher Mitgetheilten fest, dass durch die genannten Eingriffe in das Centralnervensystem die Wärmeökonomie des thierischen Organismus in der Weise gestört werden kann, dass unter Steigerung der Wärmebildung und der Wärmeabgabe der Körper seine normale Temperatur nicht mehr zu bewahren im Stande ist; die Folge davon ist unter den geeigneten Bedingungen eine über das Normale dauernd hinausgehende Steigerung derselben.

Indessen ist es noch nicht bewiesen, dass die Erhöhung der Körpertemperatur, welche oft die sogenannt krankhaften Zustände des Organismus begleitet, auf eine Functionsstörung des Centralnervensystems zurïckgeführt werden muss, wenn auch viele leider nicht ganz unzweideutige klinische Erfahrungen dafitr sprechen.

Noch viel weniger war es möglich Einsicht darin zu gewinnen, in welcher Weise die etwa der fieberhaften Steigerung der Körpertemperatur zu Grunde liegende Functionsstörung des Centralnervensystems gedacht werden müisse.

Es geht dies wohl am besten ans der Thatsache hervor, dass ein so tief in den fraglichen Gegenstand eingedrungener Forscher, wie Liebermeister, sich vor noch nicht gar langer Zeit genöthigt sah, die fragliche Functionsstörung des Centralnervensystems als Einstellung des die Körperwärme regulirenden Centrum auf einen höheren Temperaturgrad zu bezeichnen.

Von den verschiedenen Eingriffen, mittelst welcher wir experimentell bedeutende Steigerung' der Temperatur der 'Thierkörper hervorrufen können, ist die Einfiihrung putrider oder anderer sogenannt pyrogener Substanz in den Säftestrom einer der gebräuchlichsten. Von ihm kann man behaupten, dass die hier im Experiment wirkenden Ursachen der Temperatursteigerung die gleichen sind, welche, wenigstens in vielen jener Zustände spontaner (patho- 
logischer - fieberhafter) Temperatursteigertung sich geitend machen: das Wundfieber im weitesten Sinne darf wohl unbedenklich als ein durch Aufnahme pyrogener Substanz in den Säftestrom bedingtes angesehen werden.

Es ist aber noch keine thatsächliche Basis gewonnen, um z. B. nur zwischen zwei ganz entgegengesetzten Möglichkeiten, wie die Wirkung der pyrogenen Substanzen gedacht werden kann, zu entscheiden.

Diese beiden Möglichkeiten sind folgende:

1. Jene Substanzen wirken in der Weise, dass sie primär eine Functionsstörung der die Körperwärme regulirenden Theile des Centralnervensystems herbeifuihren; oder

2. Thre fiebererregende Eigenschaft beruht darauf, dass einmal (wie es aus Klebs' Untersuchungen folgt) die pyrogenen Substanzen Körper enthalten, welche den Sanerstoff zu ïbertragen d. h. oxydirend zu wirken vermögen; andererseits darauf, dass dieselben allesammt sich in fermentirendem Zustande befinden, also auch im thierischen Organismus die gleichen Processe anzuregen geeignet sind, welche in ihnen selbst vor sich gehen, und von deren Statthaben das Auftreten jener oxydirenden Substanzen in ihnen abhängt. Der Fermentwirkung ist durch die geringe Menge des angewandtep Fermentes keine Grenze gesetzt und so würde auch die verhältnissmässig bedeutende und langdauernde Wirkung geringer Mengen in den Körper eingeführter pyrogener Substanzen sehr wohl auf diesem Wege erklärbar sein.

Die im Nachfolgenden mitzutheilenden Versuche liefern einen Beitrag zur Entscheidung dieser offenbar wichtigen Frage.

Es wurden dieselben angeregt durch Erfahrungen, die bereits von Sapalski*) mitgetheilt sind.

Nach Einfuhrung von pyrogener Substanz in den Säftestrom von Thieren tritt meist eine Erhöhung der Körpertemperatur ein. In manchen Fällen ereignet es sich, dass diese Wirkung bei einem Thiere ausbleibt oder dass sogar die Temperatur, statt zu steigen, sinkt, obgleich die angewendete Substanz, wie der Erfolg an einem anderen Thiere lehrt, in so zu sagen normaler Weise wirksam ist.

Dies beobachtete Sapalski; er sah in mehreren Versuchen bei Kaninehen nach Einspritzung wirksamer pyrogener Substanz die Körpertemperatur sinken und fand sich genöthigt anzunehmen, dass dies Absinken der Temperatur dureh die im fieberhaften Zustande

*) Verhandlungen der Würzburger physikalisch-medicinischen Gesellschaft. 1872. 
enorm vermehrte Wärmeabgabe bedingt sei; denn es liess sich durch Erhöhung der das Thier ungebenden Temperatur dieselbe verhindern, ja statt ihrer eine Steigerung der Körpertemperatur erzieien.

Wir stellten die Versuche in folgender Weise an:

In allen Versuchen, in deren Protokollen nicht ausdrücklich das Gegentheil erwähnt, befanden sich die Thiere zur Vermeidung störender Einwirkung von Zugluft etc. in einem geschlossenen mit Glaswänden versehenen Raume, dessen Höhe bis zur First des giebelartigen Daches etwa $21 / 2^{\prime}$, dessen Breite $3^{1} / 2^{\prime}$, dessen Tiefe $11 / 2^{\prime}$ beträgt.

Derselbe ist ausreichend ventilirt, seine Atmosphäre wurde je nach Bedarf durch hineingebrachte Kältemischung abgekiihlt oder durch eine etwa $3^{\prime}$ unter demselben entzündete Flamme erwärmt.

Die durch die Flamme erwärmte Luft wurde durch einen entsprechenden Schornstein in den Kasten geleitet. Das Thier war gegen directe Berïhrung der durch den Schornstein zuströmenden wenig wärmeren Luft vollkommen geschützt. Aufgebunden wurden die Thiere niemals; kleinere umruhigere Thiere wurden in dünne Drathgaze locker eingeschlagen, worauf sie meist vollkommen ruhig sitzen.

Die Messungen fanden ausnahmslos im Rectum statt, das Thermometer wurde bis zu einer bestimmten Marke bei demselben Thiere stets gleich weit eingeführt. Narkotisirt wurden die Thiere niemals.

Als pyrogene Substanz wurde durch wiederholtes Filtriren fast vollkommen klar erhaltene Muskeljauche, deren mässig intensive Wirksamkeit vorher festgestellt war, oder ebensolcher doch unfiltrirter Eiter angewendet. Vor der Injection der pyrogenen Substanz wurde das Thier im gleichmässig temperirten Raume so lange beobachtet, bis seine Körpertemperatur annähernd eine Stunde 'hindurch vollkommen constant befundèn war. Eine solche Constanz stellte sich übrigens bei den Thieren unter nicht unerheblichem Sinken der Körpertemperatur meist schnell nach längstens $1 \frac{1 / 2}{2}$ bis 2 Stunden ein.

Nachdem dieselbe erreicht war, zeigten sich in dem von uns angewendeteu Raume - wie zahlreiche Controllversuche beweisen - niemals selbst bei viele Stunden hindurch fortgesetztem Aufenthalte der Thiere in demselben irgend erhebliche $d$. $h$. über 0,1 bis 0,3 hinausgehende Schwankungen.

Dass in anders construirten Wärmekasten vielleicht in Folge von grösserem oder geringerem Feuchtigkeitsgehalt der Luft oder in Folge anderer nicht genau zu übersehender Umstände die Sache sich ganz anders verhalten mag, soll nicht in Abrede gestellt werden. 
Die Temperaturen der umgebenden Atmosphäre $(15-30 \quad 0 \quad \mathrm{C}$. $=12-24 \quad 0 \quad$ R.), welche bei unseren Versuchen zur Anwendung kamen, sind übrigens so gewählt, dass sie vollkommen in den Bereich der für die benutzten Thiere Normalen fallen. Es ist um so weniger anzunehmen, dass der Aufenthalt der Thiere in der so temperirten und gut ventilirten Atmosphäre bestimmte Anomalien in ihrer Körperwärme mit sich bringt, als sich dieselben in vollkommen normaler Körperhaltung befanden.

Wenn man einem kleineren Thiere (Kaninchen, Meerschweinchen, Ratte*) eine mässig stark pyrogen wirkende Flüssigkeit unter die Haut spritzt, so iš́t Erhöhung der Körpertemperatur des Thieres nur dann die constante Folge, wenn die Temperatur der umgebenden Atmosphäre $240 \mathrm{C}$. und darüber beträgt. Ist die letztere geringer, so beginnen die Resultate schwankend zu werden, d. h. es erfolgte bald recht bedeutendes bald ganz geringes bald gar kein Steigen oder auch selbst ein geringes Sinken der Körpertemperatur; je weiter man die Temperatur der Umgebung herabsetzt, desto constanter stellt sich als Wirkung der pyrogenen Substanz der Temperaturabfall ein und bei $15-16{ }^{\circ} \mathrm{C}$. sieht man diese Folge bereits genau mit derselben Constanz eintreten wie bei der Umgebungstemperatur von $30^{\circ}$ das genau Entgegengesetzte, die (fieberhafte) Temperatursteigerung.

Es war in unseren Versuchen dieses Ergebniss ein durchaus constantes; es ist dasselbe durchans nicht bedingt durch die Individualität der Thiere und durch Menge und Beschaffenheit der zur Verwendung gekommenen pyrogenen Substanz.

Versuche $6-9,11,25,26,29,31,34,35$.

(Vergleiche auch spätere Versuche. Vergl. die Curven I. II. III.)

\section{Versuch.}

Ganz normales Kaninchen mit dem Bauch auifs Brett gebunden, hat bei $15,5^{\circ} \mathrm{C}$. Zimmertemp. - Rectumtemp. $38,0^{\circ} \mathrm{C}$. - sofort in den Wärmekasten $\left(22^{0}\right.$ C. $)$ gebracht - um 9 Uhr 30 M. V.-M.

$\begin{array}{cccc}\text { Zeit. } & \text { Temperatur des } & \text { Temperatur des } & \text { Bemerkungen. } \\ \text { 9. } 30 . & 38,0 & \text { Wärmekastens. } & \\ 9.45 . & 37,6 & 22 & \\ 10 . & 37,4 & 22 & \end{array}$

*) Unsere Versuche wurden in Bern im Sommer 1872 angestellt, es scheint sicher (die nachfolgenden Versuche werden zeigen, warum) dass die hier für das verschiedene Verhalten der Thiere in verschieden warmer Atmosphäre angegebenen Temperaturgrenzen bei Thieren zur Wirterszeit, vielleicht auch in kälteren Kli_ maten, andere sein werden in Folge der dann stärker entwickelten Behaarung der Thiere. 
$\begin{array}{ccc}\text { Zeit. Temperatur des } & \text { Temperatur des } \\ \text { Thieres. } & \text { Wärmekastens. } & \text { Bemerkungen. }\end{array}$

10. 15 . $37,4 \quad 22$

10. 30 . $37,4 \quad 22$

10. 45 . $37,4 \quad 22$

11. $37,4 \quad 22$

11. 30 . $37,15 \quad 23$

11. 45. $37,1 \quad 23$

12. $37,0 \quad 22,5$

12. 15 . $37,4 \quad 23$

12. 30 . $37,45 \quad 23$

12. $45 . \quad 37,6 \quad 23$

1. $37,65 \quad 23$

1. 15 . $37,8 \quad 23$

1. 30 . $38,0 \quad 23$

1. $45 . \quad 38,0 \quad 23$

2. $38,0 \quad 23$

2. 15 . $38,0 \quad 23$

2. 30 . 38,0 . 23

3. $38,05 \quad 23$

3. 15 . $38,1 \quad 23$

3. 30 . $38, \frac{15}{15} 23$

4. $38,2 \quad 23$

4. 15. 38,2

4. 30 . $38,2 \quad 23$

In 5 Stunden $30 \mathrm{Min}$. um $0,7^{0}$ C. über const. Temperatnr gestiegen.

\section{Versuch.}

Normales Kaninchen - ins Drathtuch gebunden - hat bei Zimmertemp. $\left(17^{0}\right.$ C.) Rectumtemp. $39,6^{0} \mathrm{C}$. - dann sofort in den Wärmekasten $\left(\begin{array}{lll}26 & 0 & 0 .\end{array}\right)$ gebracht.

$\begin{array}{lccc}\text { Zeit. } & \begin{array}{c}\text { Temperatur des } \\ \text { Thieres. }\end{array} & \begin{array}{c}\text { Temperatur des } \\ \text { Wärmeksstens. }\end{array} & \text { Bemerkungen. } \\ \text { 12. M. } & 39,6 & 26 & \\ \text { 12. } 30 . & 39,0 & 26 & \\ \text { 1. } & 35,8 & 26 & \\ \text { 1. } 30 . & 38,6 & 26 & \\ \text { 1. } 45 . & 38,4 & 26 & \\ \text { 2. } & 38,3 & 26 & \\ \text { 2. } 30 . & 38,1 & 26 & \\ \text { 2. } 45 . & 37,9 & 26 & \\ \text { 3. } & 37,8 & 26 & \text { Injection (4 O.-C.) derselben } \\ \text { 3. } 15 . & 37,7 & 26 & \text { Jauche wie in Vers. 6. } \\ \text { 3. 30. } & 37,7 & 26 & \\ \text { 4. } & 37,7 & 26 & \\ \text { 4. } 15 . & 37,7 & 26 & \\ \text { 4. } 30 . & 37,6 & 26,5 & \\ \text { 4. } 45 . & 37,5 & 26,5 & \\ \text { 5. } & 37,8 & 26 & \end{array}$




\begin{tabular}{|c|c|c|c|}
\hline Zeit. & $\begin{array}{l}\text { Temperatur des } \\
\text { Thieres. }\end{array}$ & $\begin{array}{l}\text { Temperatur des } \\
\text { Wärmekastens. }\end{array}$ & Bemerkungen. \\
\hline 5. 30 . & 38,0 & 26 & \\
\hline 5. 45 . & 38,1 & 26 & \\
\hline 6. & 38,2 & 26 & Das Thier am 5. Tage \\
\hline 6.15 & 38,4 & 26 & gestorben. \\
\hline 6. 30. & 38,5 & 26 & \\
\hline 6. 45 . & 38,6 & 26 & \\
\hline 7. 30. Abs. & 38,0 & 26 & \\
\hline 7. 45. & 38,0 & 26 & \\
\hline 8 Uhr Abs. & 38,0 & 26 & \\
\hline
\end{tabular}

Das Kaninchen, welches schon eine Injection erhalten hat, wurde ins Drathtuch gebunden. Zimmertemp. $18,5^{\circ} \mathrm{C}$. Rectumtemp. $39,0^{\circ} \mathrm{C}$. Um 8 Uhr 30 M. V.-M. in den Wärmekasten $\left(\begin{array}{lll}3 & 0 & C_{+}\end{array}\right)$gebracht.
Zeit.
Temperatur des
Thieres.
Temperatur des
Wärmekastens.
Bemerkungen.

$\begin{array}{rlll}\text { 8. } 30 . & 39,0 & 30 & \\ 8.45 . & 38,6 & 30 & \\ 9 . & 38,6 & 30 & \\ 9.15 . & 38,6 & 30 & \\ \text { 9. } 30 . & 38,6 & 30 & \text { Injection (4 C.-C.) derselben } \\ \text { 9. } 45 . & 38,6 & 30 & \text { Jauche wie in Vers. 6. } \\ \text { 10. } 30 . & 38,0 & 30 & \\ \text { 10. } 45 . & 3,0,0 & 30 & \\ \text { 11. } 15 . & 38,3 & 30 & \\ \text { 11. } 30 . & 38,5 & 30 & \\ \text { 11. } 45 . & 38,5 & 30 & \\ \text { 12. } & 38,4 & 30 & \\ \text { 12. } 15 . & 38,5 & 31 & \\ \text { 12. } 30 . & 38,7 & 30 & \\ \text { 1. } & 39,2 & 30 & \\ \text { 1. } 30 . & 39,4 & 30 & \\ \text { 2. } & 39,4 & 30 & \\ \text { 2. } 15 . & 39,45 & 30 & \\ \text { 2. } 30 . & 39,5 & 30 & \\ \text { 3. } & 39,8 & 30 & \\ \text { 3. } 45 . & 40,0 & 30 & \\ \text { 4. } & 40,0 & 30 & \\ \text { 4. } 15 . & 40,05 & 30 & \end{array}$

In 6 Stunden $15 \mathrm{M}$. die Temperatur des Thieres um $1,4^{\circ} \mathrm{C}$. über const. Temperatur gestiegen.

\section{Versuch.}

Das ins Drathtuch gebundene Kaninchen hat Rectumtemp. $38,6{ }^{\circ} \mathrm{C}$. Zimmertemp. $18,5^{\circ}$ C. - sofort in den Wärmekasten $\left(30^{\circ} \mathrm{C}\right.$.) gebracht. $10 \mathrm{Uhr}$ V.-M. 
$\begin{array}{ccc}\text { Zeit. Temperatur des } & \text { Temperatur des } \\ \text { Thieres. } & \text { Wärmekastens. } & \text { Bemerkungen. }\end{array}$

\begin{tabular}{|c|c|c|c|}
\hline 10. & 38,6 & 30 & \\
\hline 10.15 & 38,4 & $30^{\circ}$ & \\
\hline 10.30 & 38,5 & 30 & \\
\hline 10.45 & 38,4 & 30 & \\
\hline 11. & 38,3 & 30 & \\
\hline 11. 15. & 38,3 & 30 & \\
\hline 11. 30 & 38,3 & 30 & \\
\hline 11. 45 & 38,3 & 30 & Injection (31/2 C.-C.) dersel- \\
\hline 12. & 38,1 & 30 & ben Jauche wie Vers. 8. \\
\hline 12. 30. & 38,5 & 30,5 & \\
\hline 12. 45 & 38,45 & 30 & \\
\hline 1. & 38,6 & 30 & \\
\hline 1.15. & 38,7 & 30 & \\
\hline 1. 30. & 38,8 & 30 & \\
\hline 1. 45. & 39,0 & 30 & \\
\hline 2. & 38,95 & 30 & \\
\hline 2.15. & 39,1 & 30 & \\
\hline 2. 45 . & 39,3 & 30 & \\
\hline 3. & 39,4 & 30 & \\
\hline 3.15. & 39,5 & 30 & \\
\hline 3. 30. & 39,6 & 30 & \\
\hline 3. 45. & 39,5 & 30 & \\
\hline 4. & 39,6 & 30 & \\
\hline 4. 15. & 39,7 & 30 & \\
\hline 4. 30. & 39,6 & 30 & \\
\hline 4. 45. & 39,7 & 30 & \\
\hline 5. & 39,7 & 30 & \\
\hline 5.15. & 39,7 & 30 & \\
\hline
\end{tabular}

\section{Versuch.}

Im Zimmer bei $14^{\circ} \mathrm{C}$. umgebender Temperatur ausgeführt. - Das Kaninchen ins Drathtuch gebunden hat $38,7^{\circ} \mathrm{C}$.

$$
\text { Zeit. } \quad \text { Temperatur des } \quad \text { Thieres. }
$$

8 Uhr V.M. $\quad 38,7$

8. 15 . $\quad 38,5$

8. $30 . \quad 38,4$

$9 . \quad 38,2$

9. $30 . \quad 38,2$ Injection (3 C.-C. $)$ derselben

10 . 37,8

10. 15.

10. 30 . 37,5

10. 45 . 37,2

$11 . \quad 37,3$

11. 15 . 37,15

11. 45 . 36,9 . 
Zeit. Temperatur des Thieres.

12. 15 . 36,8

12. 45 . 36,9

1. 37,0

$1,30 . \quad 37,0$

Körpertemp. in 2 Stunden 15 M. nach Injection um $1,2^{\circ} \mathrm{C}$. gesunken.

\section{Versuch.}

Rectumtemp. des ins Drathtuch gebundenen Kaninchens ist $39,2^{\circ} \mathrm{C}$. Zimmertemp. 21,6 ${ }^{\circ} \mathrm{C}$. Sofort in den Kasten gebracht, welcher künstlich bis $14^{\circ} \mathrm{C}$. abgekühlt ist.
Zeit. Temperatur des
Temperatur des
Kastens.
Bemerkungen.

2. N.-M.

39,2

14

2. 15 .

38,9

14

2. 30 .

38,7

2. 45 .

38,7

14

3.

38,6

14

3. 15 .

38,5

3. 30 .

38,5

14

4.

38,5

4. 15 .

38,5

4. 45 .

38,2

5 .

38,1

14

14

14

14,5

37,95

5. 15 .

37,8

15

5. 30 .

37,5

15

5. 45 .

37,5

15

6.

37,6

15

6. 15 .

37,5

15

6.30 .

37,5

7. $37,5 \quad 15$

7. $37,5 \quad 15$

In 1 Stunde 30 M. nach der Injection um 10 C. unter const. Temperatur gesunken.

26. Versuch.

Das ins Drathtuch gebundene Kaninchen hat Rectumtemp. $39,5^{\circ} \mathrm{C}$. Sofort in den Wärmekasten bei $30^{\circ} \mathrm{C}$. gebracht. Zimmertemp. $18,5^{\circ} \mathrm{C}$. Zeit. Temperatur des Temperatur des Zeit. Thieres. Wärmekastens.

Bemerkungen.

$\begin{array}{lll}\text { 8. V.-M. } & 39,5 & 30 \\ \text { 8. } 30 . & 39,3 & 30 \\ \text { 8. } 45 . & 39,3 & 30 \\ \text { 9. } & 39,35 & 30 \\ \text { 9. } 15 . & 39,3 & 30 \\ \text { 9. } 30 . & 39,3 & 30 \\ \text { 9. } 45 . & 39,3 & 30\end{array}$




$\begin{array}{ccc}\text { Zeit. } & \begin{array}{c}\text { Temperatur des } \\ \text { Thieres. }\end{array} & \begin{array}{c}\text { Temperatur } \\ \text { Wärmekast }\end{array} \\ \text { 10. 15. } & 39,1 & 30 \\ \text { 10. 30. } & 39,2 & 30 \\ \text { 10. } 45 . & 39,4 & 30,5 \\ \text { 11. } & 39,5 & 30,5 \\ \text { 11. } 15 . & 39,6 & 30,5 \\ \text { 11. 30. } & 39,65 & 30 \\ \text { 11. 45. } & 39,8 & 30 \\ \text { 12. } & 39,9 & 30 \\ \text { 12. } 15 . & 40,0 & 30 \\ \text { 12. 30. } & 40,2 & 30 \\ \text { 12. 45. } & 40,2 & 30 \\ \text { 1. } & 40,2 & 30\end{array}$

Die Temperatur des Thieres ist in 2 Stunden $45 \mathrm{M}$. nach der Injection um 0,90 O. über const. Temperatur gestiegen.

29. Versuch.

Das ins Drathtuch gebundene Meersehweinchen hat Rectumtemp. $38,8^{\circ}$ C. Zimmertemp. $22^{\circ}$ C. Sofort in den Wärmekasten gebracht.

$\begin{array}{lccc}\text { Zeit. } & \begin{array}{c}\text { Temperatur des } \\ \text { Thieres. }\end{array} & \begin{array}{c}\text { Temperatur des } \\ \text { Wärmekastens. }\end{array} & \text { Bemerkungen. } \\ \text { 11. Y.-M. } & 38,8 & 30 & \\ \text { 11. } 15 . & 38,6 & 30 & \\ \text { 11. } 30 . & 38,6 & 30 & \\ \text { 11. 45. } & 38,5 & 30 & \\ \text { 12. } 15 . & 38,5 & 30 & \\ \text { 12. } 30 . & 38,5 & 30 & \text { Injection (1 G.-C.) Janche. } \\ \text { 1. } & 38,3 & 30 & \\ \text { 1. } 15 . & 38,6 & 30 & \\ \text { 1. } 30 . & 38,65 & 30 & \\ \text { 1. } 45 . & 38,9 & 30 & \\ \text { 2. } & 38,8 & 30 \\ \text { 2. } 15 . & 39,0 & 30 \\ \text { 2. } 30 . & 39,0 & 30 \\ \text { 3. } & 39,3 & 30 \\ \text { 3. } 15 . & 39,6 & 30 \\ \text { 3. } 30 . & 39 & 30 \\ \text { 3. } 45 . & 39,9 & 30 \\ \text { 4. } & 40,0 & 30 \\ \text { 4. } 15 . & 40,2 & 30 \\ \text { 4. } 30 . & 40,3 & 30 \\ \text { 4. } 45 . & 40,2 & 30 \\ \text { 5. } & 40,3 & 30\end{array}$

In 4 Stunden nach Injection um 1,70 C. über constante Temperatur gestiegen. Vergl. Versuch 30.

31. Versuch.

Das ins Drathtuch gebundene Meerschweinchen hat Rectumtemp. 39,50 C. Zimmertemp. 22,90 C. Sofort in den Wärmekasten $\left(30^{\circ} \mathrm{C}\right.$.) gebracht. 
$\begin{array}{ccc}\text { Zeit. Temperatur des } & \text { Temperatur des } & \text { Thieres. } \\ \text { Wärmekastens. } & \text { Bemerkungen. }\end{array}$

$\begin{array}{lll}\text { 2. N.-M. } & 39,5 & 30 \\ \text { 2. } 15 . & 39,25 & 30 \\ \text { 2. } 30 . & 39,1 & 30 \\ \text { 2. } 45 . & 39,1 & 30 \\ \text { 3. } & 39,1 & 30 \\ \text { 3. } 30 . & 38,7 & 30,5 \\ \text { 3. } 45 . & 39,1 & 30,5 \\ \text { 4. } 15 . & 39,6 & 30,5 \\ \text { 4. } 30 . & 39,95 & 30 \\ \text { 4. } 45 . & 40,40 & 30 \\ \text { 5. } & 40,75 & 30 \\ \text { 5. } 15 . & 40,9 & 30 \\ \text { 5. } 30 . & 40,9 & 30 \\ \text { 5. } 45 . & 40,9 & 30\end{array}$

30

30

30

30 Injection (1 C.-C.) derselben

30,5 Jauche wie in 30.

Die Temperatur ist in 2 Stunden 15 M. nach der Injection um $1,9^{\circ}$ C. über constante Temperatur gestiegen. Vergleiche Versuch 30 und 32 .

\section{Versuch.}

Ganz normale Ratte, 70 Gramm schwer, ist ins Drathtuch gebunden. - Rectumtemp. $37,7^{\circ}$ C. Sofort in den Wärmekasten $\left(30^{\circ}\right.$ C.) gebracht.

\section{Zeit.}

2. N.-M.

2. 15 .

2. 45 .

3. 15 .

3. 20.

3. 35 .

3. 50 .

4. 20 .

4. 50 .

5. 5 .

5. 35 .

5. 50 .

6. 20 .

6. 35 .

7. 5 .

7. 20 .
Temperatur des
Thieres.

\section{7,7}

37,3

37,3

37,3

36,9

37,8

37,9

38,05

38,6

39,11

39,6

39,8

40,35

40,5

40,45

40,5
Temperatur des

Wärmekastens.
Bemerkungen.

30
30
30
30
30
30
30
30
30
30
30
30
30
30
30
30

In 3 Stunden nach Injection um 3,20 C. über const. Temperatur gestiegen. Vergl. Vers. 33 . Ratte.

\section{Versuch.}

Ins Drathtuch gebundene Ratte von 75 Gramm Gewicht, hat Rectumtemperatur $38,5^{\circ}$ C. Sofort in den Wärmekasten $\left(\begin{array}{lll}30^{\circ} & \text { C. })\end{array}\right.$ gebracht. 
Beiträge zur Lehre von der fieberhaften Temperaturerhöhung.

Zeit. Temperatur des Temperatur des
Thieres.

$\begin{array}{llll}\text { 11. V.-M. } & 38,5 & 30 & \\ \text { 11. } 15 . & 38,6 & 30 & \\ \text { 1. } 30 . & 38,6 & 30 & \\ \text { 12. } 15 . & 37,6 & 30 & \\ \text { 12. } 45 . & 38,0 & 30 & \text { Injection } 1 / 4 \text { C.-C.)derselben } \\ \text { 1. } 15 . & 38,0 & 30 & \text { Jauche. } \\ \text { 1. } 30 . & 37,6 & 30 & \text { Während des Versuches } \\ \text { 1. } 45 . & 38,1 & 30 & \text { ganz ruhig. } \\ \text { 2. } & 38,4 & 30 & \\ \text { 2. } 30 . & 38,9 & 30 & \\ \text { 2. } 45 . & 39,3 & 30 & \\ \text { 3. } 15 . & 39,9 & 30 & \\ \text { 3. } 45 . & 40,6 & 30 & \\ \text { 4. } 15 . & 40,9 & 30 & \\ \text { 4. } 30 . & 41,0 & 30 & \text { Am 2. Tage gestorben. } \\ 5 . & 41,0 & 30 & \end{array}$

In 3 Stunden nach Injection um $3,1{ }^{\circ} \mathrm{C}$. über constante Temperatur gestiegen. Vergl. Ver's. 33. Ratte.

Auch bei Hunden zeigt sich die Höhe, bis zu welcher die Körpertemperatur nach der Jaucheeinspritzung steigt, abhängig davon ob die Bedingungen für die Wärmeabgabe von der Haut des Thieres mehr oder weniger grinstig sind; die Höhe der umgebenden Temperatur ist aber hier offenbar von weit geringerem Einfluss, wie bei jenen kleineren Thieren; sie tritt deutlich hervor, wenn die Wärmeabgabe von der Haut der Thiere durch Scheeren der Haare èrleichtert ist.

\section{Versuch.}

Der langhaarige Hund hat Rectumtemp. constant $39,8^{\circ} \mathrm{C}$. Temperatur des Kellers 14,80 C. - liegt frei und -rulig anf dem Tische. (6 Kilo schwer.)
Zeit. Temperatur des
Bemerkungen.

2 Uhr N.M. Thieres.

2. $30 . \quad 39,8$

$3 . \quad 39,8$

3. 15 . 39,6

3. $30 . \quad 40,0$

3. $45 . \quad 40,0$

4. 15 . 40,2

4. $30 . \quad 40,6$

5. 41,1

5. 1.5. 41,2

5. $45 . \quad 41,2$

$6 . \quad 41,1$

6. 30 . 41,2 
In 2 Stunden 15 M. nach Injection um $1,4^{\circ} \mathrm{C}$, über constante Temperatur gestiegen.

\section{Versuch.}

Derselbe Hund (36) wurde geschoren - hat constante Temperatur $40,0^{\circ}$ C. Temperatur des Kellers $14,8^{\circ} \mathrm{C}$.

Zeit. Temperatur des Thieres.

2. N.-M. $\quad 40,0$

3. $\quad 40,0$

3. 15 . 40,0

3. 30 . $\quad 39,7$

4. 39,7

4. $15 . \quad 40,0$

4. 45 . 40,3

5. $\quad 40,5$.

5. 15 . 40,6

5. 45 . 40,6

6. 15 . 40,6

Injection von derselben Jauche (7 C.-C.), noch in gleicher Weise wirksam.

In 2 Stunden nach der Injection Erhöhung der Temperatur um $0,60^{\circ} \mathrm{C}$.

Doch bedarf es dann noch viel niederer Temperaturgrade der umgebenden Atmosphäre um den bei dem Kaninchen etc. soviel leichter eintretenden Temperaturabfall nach der Jaucheeinspritzung zu erzwingen. Es gelang dies mit dem geschorenen Hunde erst in einem Raume (Eiskeller) dessen Temperatur $9,5^{\circ}$ betrug.

38. Versuch. (Vergl. Curve Nro. IV.)

Dieser Versuch wurde mit demselben Hande (Nro. 36. 37) im Eiskeller von $9,5^{\circ} \mathrm{C}$. Temperatur ausgeführt.
Zeit. Temperatur des
Bemerkungen.

$\begin{array}{ll}\text { 10.15. V.-M. } & 40,2 \\ \text { 10. } 30 . & 39,9 \\ \text { 11. } & 39,9 \\ \text { 11. } 30 . & 39,9 \\ \text { 12. } & 39,8 \\ \text { 12. } 30 . & 39,9 \\ \text { 12. } 45 . & 39,6 \\ \text { 1. } & 39,5 \\ \text { 1. 30. } & 39,2 \\ \text { 2. } 0 . & 39,0 \\ \text { 2. } 15 . & 38,6 \\ \text { 2. } 30 . & 38,6 \\ \text { 3. } & 33,6\end{array}$

In 2 Stunden 45 M. nach Injection Erniedrigung um 1,3 ${ }^{\circ} \mathrm{C}$. 
Die folgenden Versuche zeigen, wie wichtig auch bei den klei neren Thieren (Kaninchen, Meerschweinchen) es für das Verhalten der Körpertemperatur nach Einspritzung pyrogener Substanz ist, ob sich die Haut derselben unter für die Wärmeabgabe seitens derselben guinstigen oder ungünstigen Bedingungen befindet.

Steigert man durch ganz oberflächliches Abscheeren der Haare*) und durch Einwickeln der Thiere in mehrfache Lagen Staniol die Wärmeabgabe von der Haut derselben, so bleibt bei Temperaturgraden der umgebenden Atmosphäre, bei welchen sonst im Experiment fast ausnahmslos Steigerung der Körpertemperatur eintritt, eine solche aus**).

Vergl. Vers. 39 und 41 mit Vers. 40 und vergl, Vers. 42 mit Vers. 43. Vergl. Curve Nro. V.

\section{Versuch.}

Das wenig geschorene Kaninchen wurde in mehrere Schichten Staniol gewickelt und in den Wärmekasten $\left(26^{\circ}\right.$ C.) gebracht.

$\begin{array}{lccc}\text { Zeit. } & \begin{array}{c}\text { Temperatur des } \\ \text { Thieres. }\end{array} & \begin{array}{c}\text { Temperatur des } \\ \text { Wärmekastens. }\end{array} & \text { Bemerkungen. } \\ \text { 11. } 30 . \text { V.-M. } & 39,2 & 26 & \\ \text { 12. } & 38,35 & 26 & \\ \text { 12. } 30 . & 38,6 & 26 & \\ \text { 1. } 30 . & 38,6 & 27 & \\ \text { 1. } 45 . & 38,6 & 26 & \\ \text { 2. } 15 . & 38,2 & 26 & \\ \text { 2. } 45 . & 38,4 & 26,5 & \\ \text { 3. } & 38,6 & 26 & \\ 3.30 . & 38,6 & 26 & \\ 3.45 . & 38,6 & 26 & \\ \text { 4. } & 38,6 & 26 & \end{array}$

40. Versuch.

Kaninchen ohne Staniol bei $26^{\circ} \mathrm{C}$. im Wärmekasten.

$\begin{array}{ccc}\text { Zeit. Temperatur des } & \text { Temperatur des } \\ \text { Thieres. } & \text { Wärmekastens. } & \text { Bemerkungen. }\end{array}$

10. V.-M. $\quad 38,5 \quad 26$

10. $15 . \quad 38,4 \quad 26$

10. 30 . $38,4 \quad 26$

11. $38,4 \quad 26$ Injection von derselben

11. 15. $38,3 \quad 26$ Jauche (2 C. C.) wie 39 .

11. $38,4 \quad 26$

*) Vollständiges Scheeren scheinen diese Thiere nicht zu vertragen, wenigstens sahen wir dieselben nach dieser Behandlung ohne sonstigen schädlichen Einflüssen ausgesetzt gewesen zu sein unter stetiger Abkühlung zu Grunde gehen.

**) Zur Anstellung*dieses Versuches wurden wir durch eine Bemerknng des Herrn Professor Valentin in Bern angeregt. 


$\begin{array}{cccc}\text { Zeit. } & \begin{array}{c}\text { Temperatur des } \\ \text { Thieres. }\end{array} & \begin{array}{c}\text { Temperatur des } \\ \text { Wärmekastens. }\end{array} & \text { Bemerkungen. } \\ \text { 11. } 45 . & 38,5 & 26 & \\ \text { 12. } & 38,55 & 26 & \\ \text { 12. } 30 . & 38,9 & 26 & \\ \text { 12. 45. } & 39,0 & 26 \\ \text { 1. } 15 . & 39,2 & 26 \\ \text { 1. } 45 . & 39,4 & 26 & \\ \text { 2. } 15 . & 39,5 & 26 & \\ \text { 2. } 45 . & 39,5 & 26 & \\ \text { 3. } 15 . & 39,5 & 26 & \\ \text { 4. } & 39,5 & 26 & \end{array}$

In 3 Stunden 15 M. nach Injection 1,10 C. über constante Tem. peratur gestiegen.

\section{Versuch.}

Ganz normales weisses Kaninchen, hat Rectumtemp. 39,50 C. vor dem Scheeren - nach dem Scheeren $38,4^{\circ}$ C. Sofort in Staniol gewickelt und in den Wärmekasten bei $26^{\circ} \mathrm{C}$. gebracht, um $12 \mathrm{Uhr}$ Mittags.

Zeit. Temperatur des Temperatur des Thieres.
Wärmekastens.

2. 15 .

Bemerkungen.

2. 30 .

37,2

26

2. 45 .

37,0

26

3.

36,6

26

3. 15 .

36,6

26

3. 30 .

36,6

26

4.

36,6

4. 30 .

37,0

26,5

4. 45 .

36,9

26,5

5. 15 .

36,95

26,5

Injection von (2 C.-C.) Eiter aus dem Abscess rom Hunde; sehr wirksam.

Nur um $0,4^{\circ} \mathrm{C}$. gestiegen.

\section{Versuch.}

Das Meerschweinchen in mehrere Schichten Staniol gewickelt und in den Wärmekasten gebracht.

$\begin{array}{lccc}\text { Zeit. } & \begin{array}{c}\text { Temperatur des } \\ \text { Thieres. }\end{array} & \begin{array}{c}\text { Temperatur des } \\ \text { Wärmekastens. }\end{array} & \text { Bemerkungen, } \\ \text { 9. } 15 . & 39,4 & 24 & \\ \text { 9. } 45 . & 38,8 & 24 & \\ \text { 0. } 15 . & 38,7 & 25 & \\ \text { 0. } 45 . & 38,7 & 25 & \\ \text { 1. } & 38,7 & 25 & \\ \text { 1. } 30 . & 38,3 & 26 & \\ \text { 2. } & 38,3 & 26,5 & \\ \text { 2. } 45 . & 38,6 & 26 & \\ \text { 1. } & 38,7 & 26 & \\ \text { 1. } 15 . & 38,7 & 26 & \\ \text { 1. } 30 . & 38,9 & 26 & \end{array}$


$\begin{array}{ccc}\text { Zeit. Temperatur des } & \text { Temperatur des } \\ \text { Thieres. } & \text { Wärmekastens. } & \text { Bemerkungen. }\end{array}$

$\begin{array}{lll}\text { 1. } 45 . & 38,7 & 26 \\ \text { 2. } & 38,8 & 26 \\ \text { 2. } 30 . & 38,7 & 26\end{array}$

43. Versuch.

Das Meerschweinchen ins Drathtuch gebunden und in den Wärmekasten gebracht. (Ohne Staniol.)

Zeit. Temperatur des Temperatur des

$\begin{array}{lll}\text { 11. V.-M. } & 39,6 & \mathbf{2 6} \\ \text { 11. 30. } & 39,1 & \mathbf{2 6} \\ \text { 11. } 45 . & 39,1 & 26 \\ \text { 12. } & 39,1 & \mathbf{2 6} \\ \text { 12. } 30 . & 39,1 & \mathbf{2 6} \\ \text { 12. } 45 . & 39,2 & 26 \\ \text { 1. } & 39,8 & \mathbf{2 6 , 5} \\ \text { 1. } 30 . & 39,85 & \mathbf{2 6} \\ \text { 2. } & 39,9 & \mathbf{2 6} \\ \text { 2. } 30 . & 40,4 & \mathbf{2 6} \\ \text { 3. } & 40,5 & \mathbf{2 6} \\ \text { 3. } 30 . & 40,5 & \mathbf{2 6} \\ \text { 4. } & 40,5 & 26\end{array}$

In 3 Stunden bis $1,3^{0} \mathrm{C}$. gestiegen.

Im Gegensatz hierzu gelingt es bei diesen Thieren, wenn man die Wärmeabgabe von der Haut durch Einwickelung in dicke Lagen Watte vermindert, noch deutliche Temperatursteigerung bei Graden der Umgebungstemperatur zu erzielen, bei welchen ohne diese Maassnahme dieselbe ausbleibt oder sogar sich schon Temperaturabfall einstellt.

Vergl. Vers. 44-47 mit Vers. 3, 22, 23 und vergl. Vers. 48 und 49 mit Vers. 30, 32. - Vergl. Curve Nro. VI.

\section{Versuch.}

Ganz normales Kaninchen wurde in Watte gut gehüllt und in den Wärmekasten $\left(16^{0} \mathrm{C}\right.$.) gebracht.

$\begin{array}{ccc}\text { Zeit. Temperatur des Temperatur des } & \text { Thieres. } \\ \text { Wärmekastens. } & \text { Bemerkungen. }\end{array}$

$\begin{array}{llll}\text { 10. V.-M. } & 38,5 & 16 & \\ \text { 10. } 15 . & 38,1 & 16 & \\ \text { 10. } 30 . & 38,0 & 16 & \\ \text { 11. } & 38,0 & 16 & \\ \text { 11. } 15 . & 37,5 & 16 & \\ \text { 1. } 30 . & 38,0 & 16,5 & \\ \text { 11. } 45 . & 38,2 & 16 & \\ 12 . & 38,0 & 16 & \end{array}$


$\begin{array}{ccc}\text { Zeit. Temperatur des } & \text { Temperatur des } \\ \text { Thieres. } & \text { Wärmekastens. }\end{array}$

$\begin{array}{rrr}\text { 12. } 30 . & 38,5 & 17 \\ 1 . & 38,8 & 16 \\ 1.30 . & 38,8 & 16 \\ 1.45 . & 38,8 & 16\end{array}$

In 2 Stunden Erhöhung um $0,8^{\circ} \mathrm{C}$.

Ohne Einwickelung mit einem schlechten Wärmeleiter fiel die Temperatur des Thieres bei $16^{\circ} \mathrm{C}$.

46. Versuch.

Das in Watte gewickelte Kaninchen hat Rectumtemp. 38,90 C. Sofort in den Wärmekasten $\left(22^{\circ} \mathrm{C}\right.$.) gebracht.

Zeit. Temperatur des Temperatur des

Thieres. Wärmekastens. Bemerkungen.

8. V.-M. $\quad 38,9 \quad 22$

8. $30 . \quad 38,6 \quad 22$

8. $45 . \quad 38,6 \quad 22$

9 . $\quad 38,6 \quad 22$

9. $30 . \quad 38,6 \quad 22$

$10 . \quad 38,4 \quad 22$

10. $30 . \quad 38,6 \quad 22$

10. 45 . $38,75 \quad 22$

11. $38,8 \quad 22$

11. $15 . \quad 38,8 \quad 22$

11. $30 . \quad 39,1 \quad 22$

11. $45 . \quad 39,3 \quad 22$

12. $39,45 \quad 22$

12. $30 . \quad 39,6 \quad 23$

1. $39,6 \quad 23$

1. $15 . \quad 39,55 \quad 23$

1. $45.39,5 \quad 23$

In 3 Stunden Erhöhung über const. Temperatur um $1^{\circ} \mathrm{C}$.

\section{Versuch.}

Ganz normales in Watte gewickeltes Kaninchen hat Rectumtemp. 38,70 C. Sofort in den Wärmekasten $\left(15^{0}\right.$ C. $)$ gebracht.

$\begin{array}{ccc}\text { Zeit. Temperatur des } & \text { Temperatur des } \\ \text { Thieres. } & \text { Wärmekastens. } & \text { Bemerkungen. }\end{array}$

2. N. M. 38,2

2. 30 . 38,2

2. 45 . $\quad 38,2$

3. 15 . 38,0

3. $30 . \quad 38,2$

3. 45 . $\quad 38,4$

4. $30 . \quad 38,0$

5. $30 . \quad 38,4$

6. 15 . 38,4
15

15

15 Injection von Jauche (3

15

15

15

15

15

.16

Temperatur des Thieres nach Injection fast dieselbe geblieben. 
45. Versuch.

Das Kaninchen mit Watte umwickelt wird in den Wärmekasten gebracht $(220$ C. $)$

$\begin{array}{ccc}\text { Zeit. Temperatur des } & \text { Temperatur des } \\ \text { Thieres. } & \text { Wärmekastens. } & \text { Bemerkungen. }\end{array}$

1. N. M.

1. 1.5 .

1. 30 .

1. 45 .

2 .

2. 15 .

2. 30 .

2. 45 .

3.

3. 30 .

3. 45 .

4.

4. 30 .

5.

5. 15 .

5. 30 .
39,0

39,1

39,0

38,95

38,8

38,8

38,7

38,7

38,7

38,7

38,9

38,95

39,5

40,0

40,05

40,0
22

22

22

22,5

22 .

23

23

23

22

22,5

23

23

22

22,5

23

23

In 1 Stunde 30 M. Erhöhung um $1,3^{\circ} \mathrm{C}$.

\section{Versuch.}

Kräftiges Kaninchen, um 8 Uhr 30 M. V.-M. ins Drahttuch gebunden, hat, bei Zimmertemp. $16,5^{\circ} \mathrm{C}$., Rectumtemp. 36,2 0 C. In den Wärmekasten $\left(22^{\circ} \mathrm{C}.\right)$ gebracht.

Zeit. Temperatur des $\begin{gathered}\text { Temperatur des } \\ \text { Thieres. }\end{gathered}$

$\begin{array}{cll}\text { 8. } 30 . & 38,2 & 22 \\ 9 . & 37,5 & 22,5 \\ \text { 9. } 30 . & 37,1 & 23 \\ \text { 10. } & 36,9 & 22 \\ \text { 10. } 30 . & 36,7 & 22 \\ \text { 10. } 45 . & 36,7 & 22 \\ \text { 11. } 15 . & 36,7 & 22 \\ \text { 11. 30. } & 36,7 & 23 \\ \text { 11. } 45 . & 36,5 & 23 \\ \text { 12. } 15 . & 36,85 & 23 \\ \text { 12. } 30 . & 36,8 & 23 \\ \text { 1. } & 36,7 & 23 \\ \text { 1. } 30 . & 37,15 & 23 \\ \text { 2. } & 36,55 & 22,5 \\ \text { 2. } 15 . & 36,45 & 22 \\ \text { 2. } 30 . & 36,45 & 22 \\ 2.45 . & 36,4 & 22 \\ \text { 3. } 15 . & 36,5 & 22 \\ \text { 4. } 15 . & 36,35 & 22\end{array}$


$\begin{array}{ccc}\text { Zeit. Temperatur des } & \text { Temperatur des } \\ \text { Thieres. } & \text { Wärmekastens. }\end{array}$

$\begin{array}{lll}\text { 4. } 30 . & 36,3 & 22 \\ \text { 4. } 45 . & 36,3 & 22 \\ 5.15 . & 36,75 & 22 \\ \text { 5. } 30 . & 36,6 & 22 \\ 5.45 . & 36,65 & 22 \\ 6 . & 37,0 & 22 \\ 6.15 . & 36,95 & 22 \\ 6.30 . & 36,95 & 22 \\ 6.45 . & 37,0 & 22\end{array}$

Die Temperatur des Thieres ist mit verschiedenen Schwankungen erst nach 7 Stunden um $0,3{ }^{\circ} \mathrm{C}$. über constante Temperatur gestiegen.

22. Versuch.

Das ganz normale Kaninchen ins Drathtuch gebunden, hat Rectumtemperatur $38,5^{\circ} \mathrm{C}$. Zimmertemp. $20^{\circ} \mathrm{C}$. Sofort in den Kasten gebracht. $\begin{array}{ccc}\text { Zeit. } & \text { Temperatur des } \\ \text { Thieres. } & \text { Temperatur des } & \text { Wärmekastens. }\end{array}$

2. 15. N.-M. $\quad 38,5 \quad 15$

2. $30 . \quad 38,1 \quad 15$

2. $45 . \quad 38,2 \quad 15$

3. $38,05 \quad 14,5$

3. 15 . $38,1 \quad 15$

3. $30 . \quad 38,0 \quad 15$

3. 45 . $\quad 38,0 \quad 15$

4. 15 . $38,0 \quad 15$

4. $30 . \quad 38,0 \quad 15$

4. 45 . $37,8 \quad 15,5$

5. $37,9 \quad 16$

5. 15 . $37,8 \quad 15,5$

5. $30 . \quad 37,5 \quad 15$

5. $45 . \quad 37,5 \quad 15$

$6 . \quad 37,55 \quad 15$

6. $15 . \quad 37,5 \quad 15$

6. $30 . \quad 37,4 \quad 15$

6. 45 . $37,4 \quad 15$

7. $37,4 \quad 15$

7. 15 . $37,4 \quad 15$

In 2 Stunden nach der Injection um $0,6^{\circ}$ C. unter constante Temperatur gesunken.

23. Versuch.

Normales Kaninchen wurde ins Drathtuch gebunden - Rectumtemperatur 39,5. Zimmertemp. 22,30 C. - Sofort in den Kasten $\left(16^{\circ}\right.$ C.) gebracht.

$\begin{array}{ccc}\text { Zeit. Temperatur des } & \begin{array}{c}\text { Temperatur des } \\ \text { Thieres. }\end{array} & \begin{array}{c}\text { Wärmekastens. } \\ \text { Bemerkungen. }\end{array}\end{array}$

$\begin{array}{lll}\text { 1. N.-M. } & 39,5 & 16 \\ \text { 1. } 15 . & 39,4 & 16\end{array}$




$\begin{array}{lccc}\text { Zeit. } & \begin{array}{c}\text { Temperatur des } \\ \text { Thieres. }\end{array} & \begin{array}{c}\text { Temperatur des } \\ \text { Wärmekastens. }\end{array} & \text { Bemerkungen. } \\ \text { 1. 30. } & 39,3 & 16 & \\ \text { 1. } 45 . & 39,3 & 16 & \\ \text { 2. } 0 . & 39,1 & 16,5 & \\ \text { 2. } 15 . & 39,0 & 16 & \\ \text { 2. } 30 . & 38,9 & 16 & \\ \text { 2. } 45 . & 38,95 & 16 & \text { Injection (3 C.-C.) derselben } \\ \text { 3. } & 38,8 & 16 & \text { Jauche wie 22. } \\ \text { 3. } 15 . & 38,8 & 16 & \\ \text { 3. } 30 . & 38,8 & 16 & \\ \text { 3. } 45 . & 38,7 & 16 & \\ \text { 4. } 0 . & 38,7 & 16 & \\ \text { 4. } 15 . & 38,5 & 16,5 & \\ \text { 4. } 30 . & 38,6 & 16 & \\ \text { 4. } 45 . & 38,55 & 16 & \\ \text { 5. } & 38,6 & 16,5 \\ \text { 5. } 15 . & 38,5 & 16,5 \\ \text { 5. } 30 . & 38,5 & 16,5 \\ \text { 5. } 45 . & 38,45 & 16,5 \\ \text { 6. } & 38,4 & 16,5 \\ \text { 6. } 15 . & 38,4 & 16,5 \\ \text { 6. } 30 . & 38,4 & 16,5 \\ \text { 6. } 45 . & 38,3 & 16,5 \\ \text { 7. } & 38,3 & 16,5\end{array}$

In 3 Stunden $15 \mathrm{M}$. um $0,5^{\circ} \mathrm{C}$. unter const. Temperatur gesunken.

\section{Versuch.}

Normales Meerschweinchen wurde in Watte gut eingewickelt und sofort in den Wärmekasten $\left(\begin{array}{lll}16 & 0 & \mathrm{C} .\end{array}\right)$ gebracht.

Zeit Temperatur des Temperatur des Thieres.
Wärmekastens.

9. V.-M

Bemerkungen.

$\begin{array}{cll}\text { 9. V.-M. } & 38,9 & 16 \\ \text { 9. } 30 . & 38,8 & 16 \\ \text { 10. } & 38,6 & 16 \\ \text { 10. 30. } & 38,5 & 16 \\ \text { 11. } & 38,5 & 16 \\ \text { 11. 30. } & 38,5 & 16 \\ \text { 11. 45. } & 38,2 & 16 \\ \text { 12. } & 38,5 & 16 \\ \text { 12. } 30 . & 38,5 & 16 \\ \text { 1. } & 39,0 & 16 \\ \text { 1. } 15 . & 39,6 & 16 \\ \text { 1. } 30 . & 39,75 & 16 \\ \text { 1. } 45 . & 39,7 & 16 \\ \text { 2. } 15 . & 39,7 & 16 \\ \text { 2. } 30 . & 39,7 & 16\end{array}$

In 2 Stunden nach Injection um 1,2 ${ }^{\circ} \mathrm{C}$. Temperaturerhöhung. 
49. Versuch.

Meerschweinchen in Watte gewickelt und sofort in den Wärmekasten $\left(19^{\circ}\right.$ C.) gebracht.

\begin{tabular}{|c|c|c|c|}
\hline Zeit. & $\begin{array}{l}\text { Temperatur des } \\
\text { Thieres. }\end{array}$ & $\begin{array}{l}\text { Temperatur des } \\
\text { Wärmekastens. }\end{array}$ & Bemerkungen. \\
\hline 2. N.-M. & 38,45 & 19 & \\
\hline 2. 15 , & 38,3 & 19 & \\
\hline 2. 30 . & 38,3 & 19 & \\
\hline 3. & 38,3 & 19 & \\
\hline 3.15 . & 38,3 & 19 & Injection von Jauche $(11 / 2$ \\
\hline 3. 30. & 38,5 & 19 & C.-C.) \\
\hline 3. 45 . & 38,6 & 19 & \\
\hline 4. & 38,9 & 19,5 & \\
\hline 4. 30. & 39,5 & 19 & \\
\hline 4. 45 . & 39,9 & 19 & \\
\hline 5. & 40,0 & 19 & \\
\hline 5. 15. & 39,9 & 19 & \\
\hline 5. 45 . & 39,9 & 19 & \\
\hline 6. & 39,85 & 19 & \\
\hline 6.15 . & 39,9 & 19 & \\
\hline
\end{tabular}

\section{Versuch.}

Ganz normales Meerschweinchen ins Drathtuch gebunden. Rectumtemperatur $38,6^{\circ}$ C. Zimmertemp. $21,8^{\circ} \mathrm{C}$. Sofort in den künstlich abgekühlten Kasten gebracht.

$\begin{array}{ccc}\text { Zeit. Temperatur des } & \text { Temperatur des } & \text { Bemerkungen. }\end{array}$

10. 30 . V.-M. $\quad 38,6 \quad 16$

10. $45 . \quad 38,1 \quad 16$

11. $37,8 \quad 16$

11. $15 . \quad 37,7 \quad 16$

11. $30 . \quad 37,6 \quad 16$

11. $45 . \quad 37,6 \quad 16$

$12 . \quad 37,6 \quad 16$

12. $15 . \quad 37,3 \quad 16$

12. $30 . \quad 37,25 \quad 16$

12. $45 . \quad 37,3 \quad 16,5$

1 . $37,2 \quad 16,5$

1. $15 . \quad 37,1 \quad 16$

1. $30 . \quad 37,3 \quad 16$

1. $45 . \quad 37,0 \quad 16$

2. $36,9 \quad 16$

2. 15. $36,9 \quad 16$

2. 45. $36,7 \quad 16$

$\begin{array}{lll}3 . & 36,75 & 16\end{array}$

3. $15 . \quad 36,7 \quad 16$

$\begin{array}{lll}3.30 . & 36,5 & 16\end{array}$ 


$\begin{array}{rccc}\text { Zeit. } & \begin{array}{c}\text { Temperatur des } \\ \text { Thieres. }\end{array} & \begin{array}{c}\text { Temperatur des } \\ \text { Wärmekastens. }\end{array} & \text { Bemerkungen } \\ 3.45 . & 36,5 & 16 & \\ \text { 4. } & 36,5 & 16 & \\ \text { 4. } 15 . & 36,5 & 16 & \end{array}$

In 3 Stunden 30 M. Erniedrigung um $1,1^{\circ} \mathrm{C}$.

\section{2, Versuch.}

Das ins Drathtuch gebundene Meerschweinchen hat Rectumtemp. $39,1^{\circ}$ C. Zimmertemp. $22,2^{\circ}$ C. Sofort in den abgekühlten Kasten gebracht.

Zeit.

2. N.-M.

2. 30 .

2. 45 .

3.

3. 30 ,

3. 45 .

4. 15 .

4. 30 .

4. 45 .

5 .

5. 30 .

5. 45 .

6 ,

\begin{abstract}
Temperatur des
Wärmekastens.
\end{abstract}

Bemerkungen.

16

16

16

16

16

16

16

16

16

16

16

16

16
Injection (1 0. -C.) derselben Jauche wie in 30 .

In 2 Stunden nach der Injection um $0,8^{\circ} \mathrm{C}$. Temp.-Erniedrigung.

Wir unterliessen es den Einfluss der Farbe des Thieres auf den Gang des Versuches festzustellen.

Sehr anschaulich tritt das Gleiche, was die bisher mitgetheilten Versuche lehren, auch in folgendem Experimente hervor.

Drei Thieren von sehr verschiedener Grösse (Hund von 15,000 Gramm, Meerschweinchen von 400 Gramm, weisse Ratte von 60 Gramm Gewicht), also auch von sehr verschiedenem Verhältnisse der Grösse ihrer wärmeabgebenden Körperoberfläche zur Masse ihrer wärmeproducirenden inneren Organe, befinden sich während der ganzen Dauer des Versuches unter gleichen äusseren Verhältnissen auf einem. Tische liegend (Meerschweinchen und Ratte in diüne Drathgaze eingehüllt) in einem Raume (Keller), dessen sehr feuchte Atmosphäre $13^{\circ}$ warm ist. Sie erhalten gleichzeitig, nachdem ihre Körpertemperatur constant geworden ist, eine ihrem Körpergewicht etwa entsprechende Menge derselben Jauche eingespritzt. Während nun beim Hunde, bei welchem die Körperoberfläche bedeutend kleiner ist im Verhältnisse zur Körpermasse, die Körpertemperatur bedeutend steigt, tritt bei den beiden anderen Thieren ein starkes 
Sinken derselben ein; und dasselbe ist bei jedem der beiden Thiere um so stärker, je kleiner das Thier, d. h. je grösser verhältnissmässig_seine Körperoberfläche ist.

33. Versuch. Curve Nro. VII.

Der Versuch wurde in einem $13^{\circ} \mathrm{C}$. warmen Kellerraum, gleichzeitig mit einem Hunde von 15 Kilo Gewicht, mit einem Meerschweinchen von $400 \mathrm{Gr}$. Gewicht und mit einer Ratte von $60 \mathrm{Gr}$. Gewicht ausgeführt. Jedes von den Thieren bekommt ungefähr seinem KörperVolumen entsprechende Jaucheinjection.

Hund 15 Kilo sehwer.
Zeit. Temperatur des
Bemerkungen.
9. V.-M. $\quad 40,0$
9. $30 . \quad 40,0$
9. $45 . \quad 39,95$
$10 . \quad 39,95$
10. $15 . \quad 39,95$
10. 45 . 39,8
11. 15 . 40,0
11. 30 . 40,2
11. $45 . \quad 40,3$
12. 15 . 40,6
12. 45 . 41,0
2. 39,9
2. 15 . 41,0
2. $30 . \quad 41,3$
2. 45 . 41,35
$3 . \quad 41,35$
Das Thier ist ganz frei auf den Tisch gelegt.
Injection von Jauche (15 0. .C. $)$
In 4 Stunden $15 \mathrm{M}$. nach Injection um $1,3^{\circ} \mathrm{C}$. über constante Temperatur gestiegen. Meerschweinchen 400 Gramm schwer.
Zeit. Temperatur des Thieres.
9. 30 . $37,5 \quad$ Das Thier wurde ins Drathtuch gebunden.
$10, \quad 37,0$
10. 15 . 36,7
10. $30 . \quad 36,7$
10. $45 . \quad 36,7$
11. 15 . $\quad 35,4$
11. $30 . \quad 35,9$
12. $15 . \quad 34,7$
12. $30, \quad 34,7$
1. 34,1
2. 30 . 30,7
3. 15 . 28,5
3. $45 . \quad 27,5$
4. $\quad 27,5$
4. 15 . 27,5
Injection (1 C.-C.) derselben Jauche. 
In 4 Stunden nach Injection $\mathrm{nm} 9,20$ C. gesunken. Das Thier starb in 3 Stunden.

$$
\begin{array}{cc}
\text { Ratte } 60 \text { Gramm schweir. } & \text { (Curve Nro. 35.) } \\
\text { Zeit. } & \text { Temperatur des } \\
\text { Thieres. } & \text { Bemerkungen. }
\end{array}
$$
9. 30 .
38,0
Das Thier ist ins Drathtuch gebunden.
9. 45 .
37,4
10.
37,2
10. $15 . \quad 37,2$
10. $30, \quad 37,2$
11. 30 . 35,0
11. 45 . 32,6
12. 15 . 30,75
12. 30 . 29,95
1 . 28,5
2. 30 . 24,5
3. 15 . 24,0
3. 30 . 23,3
3. 45. 23,3 Das Thier am 2. Tage gestorben.

In 5 Stunden nach Injection um $13,9^{\circ} \mathrm{C}$. unter constante Temperatur gesunken.

Das Sinken bei dem Meerschweinchen und der Ratte kommt in diesem Versuche weit schneller zu Stande und geht zu weit höheren Graden, als wir dies sonst je beobachtet. Es ist dies wohl nicht allein die Folge der etwas niederen Umgebungstemperatur $\left(13^{\circ}\right.$ anstatt wie gewöhnlich $\left.15-16^{\circ}\right)$, sondern wohl hauptsächlich bedingt durch die bedeutende Feuchtigkeit der Luft des Kellers. Dass sich bei grösserer Feuchtigkeit der kalten Luft, welche das Thier umgiebt, die Bedingungen für die Wärmeabgabe von der Haut desselben gïnstiger gestalten können, ist klar.

Nach diesen Versuchen ist es sicher, dass es nicht richtig ist die Wirkung der pyrogenen Substanzen einfach in einer Steigerung der Körpertemperatur zu suchen. (cfr. Sapalski l. c.)

Der (febrile) Zustand, in den das Thier durch dieselben versetzt wird, äussert sich vielmehr darin, dass seine Körpertemperatur iuber den für die Verhältnisse, unter welchen es sich befindet, normalen Grad steigt oder unter denselben fâllt, je nachdem die Bedingungen für die Wärmeabgabe von seiner Haut ungünstige oder günstige sind.

Bei einer gewissen (gleichsam neutralen) Beschaffenheit der Bedingungen für die Wärmeabgabe von der Haut kann dieser (febrile) Zustand des Thieres bestehen, ohne eine Veränderung der Körpertemperatur in der einen oder andern Richtung zu veranlassen. 
Als Factoren der Wärmeabgabe von der Haut machen sich hier drei Momente geltend.

1) Die Differenz zwischen der Temperatur (der Haut) des Thieres und der Temperatur der das letztere umgebenden Atmosphäre

2) Die Bedeckung der Haut des Thieres mit die Wärmeabgabe (Strahlung und Leitung) seitens derselben erleichternden oder erschwerenden Medien.

3) Die Grösse des Thieres, d. h. das Verhältniss, welches zwischen dem Umfang seiner wärmeabgebenden Körperoberfläche und der Masse seiner wärmeproducirenden inneren Organe besteht.

Es ist dieser Zustand gleichsam labilen Gleichgewichtes ihrer Körpertemperatur, in den die Thiere durch die pyrogenen Substanzen versetzt werden, im Wesentlichen vollkommen demjenigen gleich, in welchen sie (Hunde) nach Durchtrennung des Ruickenmarkes im unteren Halstheile gerathen; es darf demgemäss wohl die oben aufgeworfene Frage nach der Art, auf welchem Wege die pyrogenen Substanzen ihre Wirkung. entfalten, als zu Gunsten der ersten von den beiden dort hingestellten Möglichkeiten entschieden angesehen werden, d. h. die pyrogenen Substanzen wirken auf die Wärmeökonomie des thierischen Körpers, indem sie primär eine Functionsstörung der derselben vorstehenden Theile des Centralnervensystems hervorrufen.

Welcher Art ist nun aber diese Functionsstörung der betreffenden Theile des Centralnervensystems? Handelt es sich um einen Reiz- oder Lähmungszustand derselben?

Am wahrscheinlichsten ist es wohl, dass es sich hier um Läh. mungszustände handelt. Es spricht dafiur das ïbereinstimmende Verhalten der Thiere im Fieber und im Rückenmarksversuch, es stimmen auch mit einer solchen Annahme die Erfahrungen, welche die Pathologie für das Verständniss des Fiebers an die Hand giebt. Die letzteren sind ja der Art, dass Virchow*) auf Grund dieser Erfahrungen schon vor längerer Zeit die Ursache des Fiebers in einem lähmungsartigen Zustande bestimmter Theile des Centralnervensystems giaubte annehmen zu miissen.

Dieser lähmungsartige Zustand der betreffenden Theile des Centralnervensystems bedingt - anders sind die Resultate der Fieberversuche gerade so wenig verständlich, wie die der Rückenmarksversuche - gleichzeitig eine Steigerung der Wärmeabgabe gerade so gut, wie eine Steigerung der Wärmebildung, und es ist keineswegs die Steigerung der Wärmeabgabe allein die Folge der gestei-

*) Handbuch der spec. Pathologie u. Therapie Bd. I. 
gerten Wärmeproduction ebensowenig wie letztere etwa ausschliesslich die Folge von ersterer ist. Die vermehrte Wärmeabgabe ist nun aller Wahrscheinlichkeit nach bedingt durch vasomotorische Phänomene; hierauf weist der bekannte Einfluss der Rückenmarkstrennung auf die feineren Gefässe der Haut und die Abkuhlung der Thiere hin. *)

Da liegt es dann wohl nahe auch die gleichzeitig im Fieber statthabende gesteigerte Wärmeproduction auf vasomotorische $\mathrm{Phä-}$ nomene zurïckzuführen. Dass ebenso - freilich in geringerem Grade - wie nach Rückenmarksdurchtrennung anch im Fieber erbebliche Circulationsanomalien in den inneren (wärmeproducirenden) Organen auftreten, ist mindestens sehr wahrscheinlich; wie sich dieselben gestalten, ist vorläufig nicht zu übersehen; dass dieselben zu Steigerung der Wärmebildung führen können, ist nicht zu bestreiten.

Es liessen sich hier vielerlei Möglichkeiten erörtern, von denen sich indessen keine sicher begründen lässt.

Immerhin werden wir, so lange es irgend gestattet ist anzunehmen, dass die gesteigerte Wärmeproduction die Folge des gleichen vasomotorischen Phänomens ist, das wir auf der andern Seite als Ursache der vermehrten Abkühlung wirken sehen, auf die Annahme der Wirkung direct trophischer Vorgänge verzichten.

Die hier mitgetheilten Thatsachen und die darauf zu gründenden Schliusse werden auch in der Pathologie des Menschen da zur Geltung kommen müssen, wo sogenannte fieberhafte Zustände, bedingt durch. die Aufnahme pyrogener Substanz; vorliegen; wie weit die Grenze der letzteren zu ziehen ist, kann noch nicht sicher bestimmt werden.

Das wichtigste Ergebniss in dieser Richtung scheint dann dies zu sein, dass das (septische) Fieber in der Hauptsache ein vasomotorisches Phänomen ist, welches bedingt wird durch eine Functionsstörung bestimmter Theile des Centralnervensystems. Es entfaltet sich dieses vasomotorische Phänomen nach zwei Seiten und fuhrt einerseits durch Beeinflussung der Hautgefässe (Erweiterung derselben) zur gesteigerten Wärmeabgabe, andererseits durch Beeinflussung der Circulation in den inneren Organen zur vermehrten Wärmebildung.

So werden zwei Factoren entfesselt, die sich nun gleichsam um die Beherrschung des Resultates streiten. Ob letzteres Steigerung der Körpertemperatur ist oder nicht, hängt davon ab, welcher von beiden Factoren die Oberhand gewinnt. Es ist kein Zweifel,

*) Vergl. besonders T sches chichin 1. c. pag. 157. 
dass auch bei wirklich fieberhaften Zuständen des menschlichen Körpers normale oder subnormale Temperatur desselben bestehen kann.

Die Wichtigkeit der mehr äusserlichen Umstände, welche darauf Einfluss ausiuben können, dass der eine oder andere jener beiden streitenden Factoren die Ueberhand gewinnt (und welche in obigen Mittheilungen so ausführlich behandelt sind) muss anch bei der Beurtheilung solcher Krankheitszustände am Menschen festgehalten werden - gewiss vielen Pathologen bereits geläufige oder wenigstens willkommene Anschauungen.*)

Sicher wirken aber am Menschen diese mehr äusserlichen Umstände (Temperatur der Umgebung - Bedeckung der Haut des Kranken etc.) nicht in der geradezu maassgebenden Weise, wie bei kleineren Thieren. Schon Hunde sind, wie wir sahen, den Einflüssen dieser Umstände, wegen ihrer bedeutenden Körpermasse, weniger unterworfen, als Kaninchen etc.; noch viel unabhängiger wird zweifellos der noch erheblich grössere Mensch denselben gegenuiber sein.

Weiter ist noch ein Moment festzuhalten, ohne dessen Berücksichtigung es nicht möglich sein wird die Auffassung des (selbst. nur des septischen) Fiebers als vasomotorisches Phänomen durchzuführen:

Wir wissen aus den Versuchen der Physiologie, dass es durch bestimmte Eingriffe - Reizung bestimmter Nerven, Hervorrufung von Dyspnoe etc. - gelingt, das sogenannte vasomotorische Centrum in Erregungezustand zu versetzen.

Die Contraction der feineren Gefässe, die die Folge dieser Erregung des sogenannten vasomotorischen Centrums ist, ist aber keineswegs in allen Organen unter solchen Umständen gleich stark; die neneren, namentlich die aus Ludwig's Laboratorium hervorgegangenen Untersuchungen zeigen sicher, dass sich die verschiedenen Gefässprovinzen bei Reizungen des gemeinschaftlichen Innervationscentrum in hohem Grade von einander unabhängig erkalten.

Diese Unabhängigkeit wird sich zweifellos auch in dem vaso_ motorischen Phänomen geltend machen, welches die Folge der Einwirkung des Giftes der putriden (pyrogenen) Substanzen (Sepsin - Sehmiedeberg und Bergmann) ist.

Es braucht also nicht stets die Störung in der Innervation der Hautgefässe und der der Gefässe der imeren Organe im bestimmten

*) Cfr. W underiich, Die Eigenwärme in Krankheiten. I. Auflage 1868. Pag. $131 \mathrm{ff}$. 
stets gleichen Verhältniss zu stehen. Dies Verhältniss ist wesentlicher einseitiger Abänderung fähig und solch einseitige Abänderung wird wesentlich bedingend sein für das Verhalten der Körpertemperatur.

Die Wirkungen der kalten Bäder zeigen sich (nach Fiedler's interessanten Mittheilungen ${ }^{*}$ ) z. B. als ein Mittel, eine solche bedeutende einseitige and sehr wirkungsvolle Abänderung des zwischen der Cireulation in den inneren Organen und der in der Haut beim Fieber bestehenden Verhältnisses hervorzubringen.

Ausdrücklich sei hier dann noch hervorgehoben, dass es keineswegs unsere Absicht ist, das Mitspielen von Erregungszuständen bestimmter zur Wärmeökonomie in Beziehung stehender Hirntheile beim Fieber und selbst bei dem zunächst behandelten septischen Fieber in Abrede zu stellen. Es wirden ja im Eingang dieser Arbeit die interessanten Versuche Heidenhain's in dieser Richtung mitgetheilt; die klinische Erfahrnng bietet in dem schnell vorübergehenden Schüttelfrost mit bedeutender Temperatursteigerung, wie ihn hier die Malaria (Intermittens) - dort die Aufnahme pyrogener Substanz in das Blut (Septicämie) - dort eine Schleimhautreizung (Urethralfieber - Gallenstein - Nierensteine) hervorruft, häufig Fieberzustände dar, die sich jenen Verstuchen anreihen.

Eindeutig zwar sind diese Thatsachen allesammt nicht, wissen wir doch dureh Goltz, Bernste in etc, dass Reizung bestimmter centripetaler Nerven nicht selten Erweiterungen der kleineren Gerässe bestimmter Gefässbezirke, d. h. nichts Anderes als eine vasomotorische Reflexlähmung hervorruft.

Wie dem auch sei, alle diese Fragen, wie die ganze Lehre rom Fieber werden einer weiteren Erörterung viel besser zugänglich, wenn wir festhalten, dass das Fieber ein central bedingtes vasomotorisches Phänomen im oben auseinandergesetzten Sinne ist, and für solehe Auffassung desselben liefern die hier mitgetheilten Versuche über das durch putride Substanzen bei Thieren hervorgerufene einen sicheren Anhalt.

Wir brauchen nicht daran zu erinnern, dass die Auffassung des Fiebers als vasomoțorisches Phänomen schon vor längerer Zeit von Traube $e^{* *}$ gelehrt wurde.

Wir dürfen gemäss den ausserordentlich häufigen Discussionen, lie über jene „Hypothese“ geführt wurden, als klar voraussetzen,

*) Archir der Heilkunde von Wagene r. Bd. XI.

**) Zur Fieberlehre. Gesammelte Beiträge zur Pathologie. Cfr. Bd. II. 'ag. 637. Allg. medicin-Cent.-Zeitung 1863. 
in wie weit und worin sich die hier entwickelte Anschauung ron der älteren Traube'schen unterscheidet.

Nur dies sei hier bemerkt, dass Traube auf Grundlage seiner Hypothese im Wesentlichen die pathologische Wärmeretention als Ursache der im Fieber gesteigerten Körperwärme ansehen musste; soweit seine Anschaung vom Grundrorgange beim Fieber steht oder fallt mit dieser Consequenz, soweit halten auch wir dieselbe für widerlegt.

Königsberg and Bern, Anfang Februar 1873.

Tabellarische Zusammenstellung der Versuche mit Eiter- und Jaucheinjection und nachfolgender Temperaturveränderung bei verschiedenen Temperaturen der Umgebung.

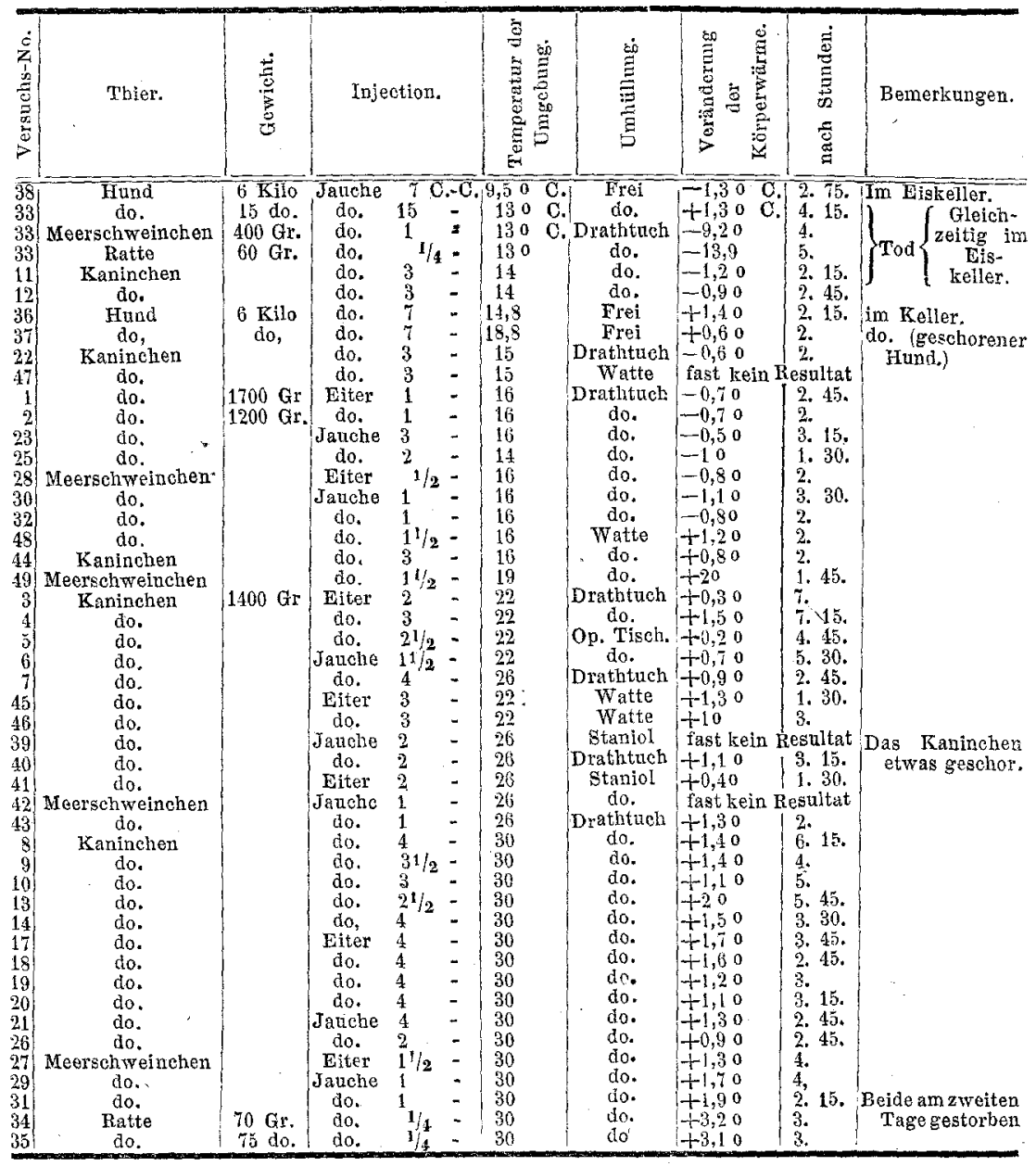

